\title{
Récrire l'histoire : théâtre et révolution dans An tan révolisyon de Maryse Condé
}

https://doi.org/10.29173/af29440

(c) $($ ) $\odot$

Fély Catan

Fely_Catan@pitzer.edu

Pitzer College, USA

Résumé. Cet article s'intéresse à la représentation de l'histoire dans le théâtre radicalement contestataire de Maryse Condé. Alors que l'histoire et la mémoire sont convoquées par le discours officiel pour glorifier les valeurs républicaines et la Révolution qui les a produites, la dramaturge s'attèle à déconstruire cette histoire positiviste en proposant une perspective créocentrique résolument tournée vers le devenir des peuples caribéens. Nous analysons ainsi la manière dont An tan révolisyon mobilise le passé pour évoquer le monde présent tout en développant un propos ambigu face aux nationalismes antillais.

Mots clés : Caraïbes; théâtre; histoire; révolution; études postcoloniales

\begin{abstract}
This article examines the representation of history in Maryse Condé's radical protest theater. While official discourse uses history and memory to glorify republican values and the Revolution that produced them, Condé seeks to deconstruct this positivist history by offering a Creocentric perspective resolutely turned towards the future of Caribbean peoples. Thus, we analyze the way in which An tan revolisyon mobilizes the past to address the present world while developing an ambiguous discourse visà-vis Antillean nationalisms.
\end{abstract}

Keywords: Caribbean, theater, history, revolution, postcolonial studies

A vec An tan révolisyon (dorénavant $A T R$ ), Condé aborde les thèmes devenus récurrents dans la production artistique caribéenne : l'émancipation, la révolution, la libération nationale, la résistance, etc. Rappelons qu'en 1946 la Guadeloupe et la Martinique firent le choix d'être pleinement rattachées à la France en devenant des Départements français d'outre-mer (DOM); tandis que 
le reste du « Tiers-Monde » allait opter pour l'indépendance dans les décennies qui suivirent. Cette œuvre est produite à un moment charnière de l'histoire antillaise de l'ère de la post-départementalisation (commémoration du bicentenaire de la Révolution française et lutte armée indépendantiste). Cependant, il serait réducteur d'y voir un pamphlet politique en faveur de l'indépendance ; cela serait ignorer l'ironie inhérente à l'œuvre condéenne.

Je propose, dans cette étude, d'explorer le contexte historique de l'écriture de cette pièce et les conditions matérielles de sa production afin d'en saisir au mieux la portée intellectuelle. En effet, ATR s'inscrit dans le débat foisonnant des années 1980-1990 autour de l'identité antillaise où s'illustrèrent les créolistes, Edouard Glissant mais aussi des historiens tel que Louis Sala-Molins. Dès lors, le travail analysera les tensions, divergences ou convergences avec ces interlocuteurs implicites (dont l'État français reste le privilégié) selon quatre axes. Tout d'abord, je me pencherai sur la manière dont Condé envisage le travail de l'histoire. Ensuite, nous aborderons l'analyse scénographique et le jeu d'acteurs. La troisième partie s'intéressera à l'appel pour une solidarité caribéenne. Enfin, nous traiterons de la question de la mémoire dans le contexte spécifiquement antillais.

Je démontrerai comment la mobilisation de registres divers (tantôt ironique, tantôt dramatique) permet d'invalider toute tentative péremptoire de designer une solution toute faite pour remédier au mal-être des peuples antillais. L'ironie chez Condé est avant tout une stratégie pour annihiler toute tentative de réduction de sa pensée.

\section{UNE CERTAINE VISION DE L'HISTOIRE}

$A T R$ est une pièce en trois parties qui relate l'épopée insurrectionnelle des peuples antillais à la fin du XVIIIe siècle et au début du siècle suivant. Cette « fresque historique en trois époques » telle que la désigne l'auteure dans le sous-titre de sa pièce, s'inspire largement de l'œuvre d'Ariane Mnouchkine, 1789 et 1794, pensée en deux mouvements. Si Maryse Condé adopte la forme de la fresque historique à la manière de Mnouchkine, elle y ajoute une troisième période dramatique intitulée « 1802 » pour contester l'historiographie officielle qui néglige délibérément les évènements révolutionnaires survenus aux Antilles (et notamment en Guadeloupe) suite au rétablissement de l'esclavage par Napoléon.

Pour les historiens occidentaux, la représentation du passé passe par l'écriture. Ils assument pour ainsi dire leur fétichisation du signe écrit. Chez Michel de Certeau, c'est par exemple déjà assumé dans le titre L'Ecriture de l'histoire. De plus, le débat se cristallise autour de la figure de l'historien qui naît en tant que tel au XIXème siècle (entendu ici comme historien moderne). Condé pulvérise ces conventions tacites sur la façon de « faire de l'histoire » et évacue un débat rébarbatif : elle refuse d'écrire l'histoire et choisit au contraire d'en faire un spectacle. Ainsi, comme la plupart des pièces de théâtre issues du répertoire antillais, cette œuvre est d'abord conçue pour être adaptée scéniquement (et non pensée initialement pour la publication, et donc pour l'écriture).

C'est parce que l'Histoire leur a toujours nié cette agencéité que les peuples caribéens doivent se l'approprier en mettant au centre de l'écriture historique des personnages autrefois marginaux (dont le nègre marron incarne la figure de proue). Dans son Discours antillais, Edouard Glissant explique que l'écriture de l'histoire ne peut être laissée aux seuls historiens ; la tâche est telle que les écrivains doivent suppléer aux ressources de l'historien. En réalité, on perçoit une certaine méfiance face au travail de

Alternative francophone

https://journals.library.ualberta.ca/af/index.php/af 
l'historien et Glissant estime l'écrivain plus apte à cerner la complexité de l'histoire antillaise dans toutes ses dimensions (y compris spirituelles et mystiques). ${ }^{1}$

À travers le personnage du conteur, Zéphyr ; Condé relève le défi lancé par Glissant. De fait, la pièce met l'oralité de la culture antillaise à l'honneur. En effet, l'Histoire nous est relatée par le conteur qui se substitue pour l'occasion à l'historien. Zéphyr est donc le personnage central de la pièce, c'est lui qui impose son rythme et nous transmet ses émotions à travers sa performance de conteur. La performance du conteur antillais s'apparente largement à celle du comédien, en ce qu'ils établissent tous deux un rapport entre la parole et la voix, mais aussi avec le corps et la gestuelle. Dans Théâtres des Antilles : Traditions et scènes contemporaines, Stéphanie Bérard commente: « En faisant du conteur le porte-parole de l'Histoire, les dramaturges antillais exploitent donc la théâtralité de la parole contre qui oscille entre vérité et mensonge, entre réalité et illusion. » (127). Chez Condé, le conteur se caractérise par son ambiguïté. Il oscille entre affabulation et vérité, compassion et jalousie, entre rire et larmes.

Si l'histoire fascine la dramaturge, celle-ci ne doit cependant être prise (trop) au sérieux et sombrer dans des principes dogmatiques, de peur de reproduire les travers de l'historiographie traditionnelle. Dans son article « L'ironie comme stratégie dramatique dans La tragédie du roi Christophe d'Aimé Césaire et An Tan Revolisyon de Maryse Condé », Christiane Makward analyse la dérision et l'ironie qui débordent l'œuvre de Condé comme la volonté d' « empêcher ses lecteurs de l'enfermer dans un certain esprit de sérieux de type 'postcolonial', dans ce 'devoir de mémoire' auxquels la plupart des romanciers de la Caraïbe nous ont accoutumés. » (35).

ATR résulte d'une commande officielle du Conseil régional de la Guadeloupe à l'occasion des célébrations du bicentenaire de la Révolution française en Guadeloupe. Cette production est fondamentale dans le parcours de l'auteure, car pour la première fois, elle produit un texte qui tient compte des possibilités de la mise en scène. ${ }^{2}$ Le budget considérable dont Condé dispose lui permet de s'inspirer librement du théâtre dialectique brechtien pour présenter sa vision critique de l'histoire. ${ }^{3}$ Mise en scène par Sonia Emmanuel, la représentation eut lieu les 11 et 12 novembre 1989, au Fort Fleur-d'Epée ; lieu hautement symbolique dans l'histoire guadeloupéenne, car c'est sur ce site que le gouverneur Victor Hughes dérouta l'envahisseur anglais grâce au renfort déterminant des esclaves noirs à qui il avait promis la liberté en retour.

La pièce fut un véritable succès populaire, comme le souligne Makward, avec un millier de spectateurs à guichets fermés pour chacune des représentations. En réalité, il semble que les autorités elles-mêmes

\footnotetext{
${ }^{1}$ Glissant explique: "La où se joignent les histoires des peuples, hier réputes sans histoire, finit l'Histoire. (Avec un grand H.) L'histoire est un fantasme fortement opératoire de l'Occident, contemporain précisément du temps où il était le seul à 'faire' l'histoire du monde." (227). Et un peu plus loin il ajoute: "A ce stade, l'Histoire s'ecrit avec un grand H. C'est une totalité qui exclut les histoires non concomitantes de celle de l'Occident." (243).

${ }^{2}$ Ainsi que le rapporte Alvina Ruprecht (10).

${ }^{3}$ Le théâtre épique de Brecht se caractérise par la distanciation qu'il instaure entre le spectateur et la pièce de théâtre (à la différence du théâtre de la tragédie classique qui souhaite provoquer l'identification et la catharsis). Cette distanciation a pour but d'initier une réflexion sur le caractère illusoire du théâtre et invite au questionnement et à l'analyse. Une des méthodes brechtiennes pour générer cette distanciation consiste en la fragmentation de la trame linéaire du spectacle. Condé s'inspire de ce procédé avec la multiplication des personnages et la polyphonie de discours (la foule représente un chœur et le personnage de Zéphyr rappelle constamment la nature meta du théâtre). On peut aussi citer le rôle du décor et des costumes dans le théâtre de Brecht pour renforcer cette distanciation. Condé souhaite un décor démesuré ainsi que des costumes grotesques pour renvoyer à la nature carnavalesque de l'histoire.
} 
n'avaient pas envisagé un tel engouement et, face à un phénomène qui les dépassait (et les inquiétait), elles préférèrent interrompre l'aventure. ${ }^{4}$ À ce propos, la notion de « event-ness » qu'Erika Fischer-Lichte définit dans The Routledge Introduction to Theater and Performance Studies éclaire sur les enjeux de cette décision plutôt drastique. La représentation théâtrale en tant que «performance » est par nature unique et imprévisible. Une fois lancée, cette performance n'est plus contrôlée ni contrôlable et il s'opère alors un transfert de pouvoir que les autorités finirent par craindre. Fischer-Lichte explique :

\begin{abstract}
A performance is an event in so far as no individual participant controls it completely. This is true not only because of the bodily co-presence of actors and spectators, but also because of the specific of presence through which phenomena emerge and how meaning is constituted from this phenomena. To the extent that phenomena appear present in especially intensive ways, they separate from their context and appear to exist in and for themselves. The phenomena occur for those who perceive them; we can say that their perception happens to a person. The perceiving subject is overcome by an oscillation between two different orders of perception, and is placed in a state of liminality. (41-42)
\end{abstract}

Selon Makward, dans l'œuvre de Condé, la dimension didactique (histoire, politique, travail de mémoire) demeure substantielle (40). À la lumière de la citation ci-dessus, nous pouvons avancer que cette affirmation n'est qu'en partie vraie. En effet, à cause du caractère unique et éphémère de la représentation théâtrale (« event-ness »), Condé sait qu'elle doit frapper fort pour marquer les esprits. La dramaturge entre dans le jeu subtil de dire sans dire tout en disant. Elle propose de lever le voile sur le refoulé historique en posant des questions qui fâchent. Dans un entretien accordé à Makward, Condé confessa que le théâtre était, selon elle, la meilleure façon de s'adresser au public. C'est par Zéphyr (alter ego de la dramaturge) que Condé interpelle ses compatriotes guadeloupéens, incités à réfléchir à une Révolution qui pour eux signifia la continuation plutôt que la rupture.

Glissant revient très largement sur l'aliénation historique des habitants de la Guadeloupe et de la Martinique. En les qualifiant de sociétés de non-histoire, Glissant affirme le rôle vital que l'histoire joue sur un peuple. De fait, il observe que "Pour un peuple qui ne s'exprime pas, pour un peuple mentalement asservi, il n'y a que la non-histoire : l'absence à toute décision et à toute maturation qui le concernent." (172). Il argumente : «Parce que le temps antillais fut stabilisé dans le néant d'une non-histoire imposée l'écrivain doit contribuer à rétablir la chronologie tourmentée, c'est-à-dire à dévoiler la vivacité féconde d'une dialectique réamorcée entre nature et culture antillaises. » (228).

Condé s'inscrit dans cette vision de l'histoire mais à la différence de Glissant, elle s'autorise davantage de libertés quant au rétablissement de « la chronologie tourmentée » comme en témoignent les nombreux anachronismes qui jalonnent la pièce. L'histoire doit être un moyen, non une fin : elle doit éclairer le présent et mener vers la vraie liberté. ${ }^{5}$ Comme l'a remarqué Nesbitt, Condé craint la récupération bourgeoise de l'histoire avec sa tendance à la muséification et à la commémoration outrancière. Ses

\footnotetext{
${ }^{4}$ Ruprecht commente: «Le résultat fut tellement puissant en 1989 que le financement a été retiré et le spectacle n'a pas pu continuer sa tournée en Martinique et en Métropole car le conseil régional a compris la nature extrêmement subversive de ce spectacle et l'appel à la contestation qui ressortent de discours politiques cités textuellement à partir des documents d'archives. » (10).

${ }^{5}$ Nick Nesbitt recueille les propos de Condé lors d'un entretien : « To know one's past, to dominate it, to know it in its reality without making of it an object of backwards-looking veneration, is one of the conditions of freedom » (Voicing memory 201).
}

Alternative francophone

https://journals.library.ualberta.ca/af/index.php/af 
anachronismes répétés désacralisent la linéarité téléologique du grand récit historique. Par exemple, une didascalie indique : « deux colons blancs portant des casques de footballeurs états-uniens ».

Les références à notre monde moderne renvoient pour la plupart à la société de consommation (capitaliste et américanisée) qui caractérise les relations de dépendance entre la métropole et les DOM. Le spectateur est poussé à adopter un rôle actif dans cette auto-analyse de peur de n'être lui aussi ridiculisé :

[Quand j'étais petit, j'avais écrit sur mes cahiers : « Je veux être Victor Hugo ou rien. » Vous imaginez cela ? Moi, petit nègre de rien du tout voilà quels étaient mes rêves. (Silence, puis il éclate de rire) Vous m'avez cru? Je vous ai bien eus! Vous savez, quand j'ai fermé les yeux, Victor Hugo était loin, bien loin d'ouvrir les siens. Et puis, de mon temps, on ne rêvait pas de poésie, de littérature, de toutes ces bêtises-là. On rêvait de liberté. Pas comme vous qui rêvez de B.M.W ou de magnétoscopes ou de vacances à Caracas! Non! De liberté. Vivre debout. Debout comme un pié-bwa !] (ATR 59-60)

Dans Non-sovereign Futures : French Caribbean Politics in the Wake of Disenchantment, l'anthropologue portoricaine Yarimar Bonilla retrace l'histoire des luttes syndicales en Guadeloupe depuis la départementalisation en se focalisant plus particulièrement sur la grève historique de 2009. Elle évoque entre autres la façon dont l'histoire est vécue et ressentie dans ce contexte insulaire et (post)colonial comme un entrelacement de temporalités :

$[\ldots]$ history is experienced as a spiral of events, spinning around a shared space and place, encompassing the landscape, and saturating it with the weight of that which came before. What becomes important is not the sequence of events - what happened and when — but the spatiality of history - what happened here. As a result, the past is ordered, not through a chronology of events, but through a thematic spiral that spins through the past, present, and future of a shared space. (145)

Dans la pièce de Condé, les évènements reproduisent cette structure spiralique de l'histoire avancée par Bonilla. Zéphyr fonctionne à la manière de l'oracle de la tragédie classique, il incarne la vision prophétique du passé :

CONTEUR : «Oui, vous avez déjà vu ces scènes de désespérance et de deuil. Pareilles au fil des années et de nos jours encore. Des hommes mourants, des hommes enfermés dans les quartiers de haute sécurité des prisons pour la liberté de leur peuple, des femmes en pleurs, des orphelins. Vous les avez vus, vous les avez vus. Vous les voyez encore aujourd'hui. Notre histoire n'est faite que de recommencements...» (57)

Cette réplique fait directement allusion à l'histoire de la lutte indépendantiste en Guadeloupe et dut résonner très fort dans ce public averti. En effet, à partir des années 1980, les plus radicaux parmi les mouvements indépendantistes se tournent vers la lutte armée afin de faire entendre leurs voix. Ces militants prônent la guérilla et se regroupent dans le Groupe de libération armée (GLA), le Mouvement populaire pour la Guadeloupe indépendante (MPGI), ou encore l'Alliance révolutionnaire caribéenne (ARC). Luc Reinette est la figure emblématique de cette mouvance ; il devient un héros populaire à la fin des années 1980 après s'être évadé de sa prison de Basse-Terre où il purgeait une peine pour sa condamnation suite à des actes terroristes qui lui étaient imputés. Cette évasion fit de lui un nouveau nègre marron dans l'imaginaire populaire : il échappa à la justice trois années durant avant d'être finalement arrêté en 1987 à Saint-Vincent où il était allé chercher l'asile politique ${ }^{6}$.

Suite à sa capture, Reinette fut condamné à trente ans de réclusion. En 1989, François Mitterrand lui accorda la grâce présidentielle. Condé profite de cette « coïncidence » pour détourner la commémoration et animer le débat de la libération nationale. Ainsi, Zéphyr-Condé connecte passé et monde contemporain, les combattants de la liberté d'antan à ceux d'aujourd'hui. Bien qu'elle n'ait jamais dissimulé son soutien à

\footnotetext{
${ }^{6}$ Non-Sovereign Futures, p. 34-35.
} 
la cause indépendantiste (elle se présenta d'ailleurs aux élections de 1992 en Guadeloupe); Condé ne fait pas de son art un outil de propagande politique. Derrière cet appel au changement, les contours de la révolution prônée dans $A T R$ restent flous... Condé le dira elle-même : «A writer does not have a political program. If writers come out with programs, they are politicians, not writers. » (Conversations with Maryse Condé 90).

\section{SCÉNOGRAPHIE ET JEUX D’ACTEURS : UN THÉÂTRE QUI DÉSTABILISE}

L'espace scénique tripartite remet en question l'idée d'une république (et d'une nation française) une et indivisible tout comme l'a fraîchement proclamée la Révolution. Visuellement, le spectateur constate l'éclatement spatial qui met à jour trois « zones » distinctes. En outre, on peut également interpréter cet éclatement comme un questionnement sur l'unité, l'unicité du discours officiel historique qui n'accepte d'autre version de l'histoire française (et de surcroît s'agissant de la Révolution française). Makward a décrit la scène de Fort Fleur d'Épée de la manière suivante :

La scénographie reposait donc sur deux « podiums » ou aires de jeux surélevées avec un certain nombre de praticables et les rampes au sommet du Fort. On observe en effet un espace surélevé, large chemin de ronde fermé par un parapet d'un mètre de hauteur, avec deux rampes d'accès (pour les canons et les véhicules) de part et d'autre d'une aire pavée centrale au-delà de laquelle les spectateurs se disposaient en arc-de-cercle. Il faut imaginer les aires de jeu (désignées dans le texte comme podium 1 ou de gauche, et 2 ou de droite) comme étant assez spacieuses pour accommoder par exemple la charrette tirée par des esclaves qui ouvrait le spectacle : il en descendait deux hommes forts, deux colons blancs portant des casques de footballeurs états-uniens... Dans une autre scène, sur l'aire de droite, c'était le trône de Louis XVI, avec d'une part ses conseillers et ministres, et d'autre part des figurants du peuple qui cernaient le monarque. (15)

En fragmentant l'histoire de la Révolution en différentes unités spatio-temporelles, c'est le mythe universaliste et atemporel de cette dernière qui est remis en question. Car la Révolution de 1789 n'a pas eu lieu pour tout le « peuple de France ». Mireille Rosello le souligne dans son livre Littérature et Identité créole aux Antilles: [...] la révolution marquait une nouvelle origine, l'avènement de ce que les postmodernes appellent un metarécit, un mythe accompagné de tout un cortège de valeurs inattaquables (abolition des privilèges, ère de la liberté, de l'égalité, de la fraternité), valeurs qui, à force de se voir imprimées sur les billets de banque, sont plus ou moins devenues des clichés. (113-114).

De la même façon, dans Voicing memory : History and subjectivity in French Caribbean literature Nick Nesbitt note que la pensée des Lumières est intrinsèquement et fondamentalement contradictoire. Elle définit le principe d'humanité au moment même où elle exclut tout un pan de l'humanité de ce principe abstrait, contredisant la notion même d'universalité. Il s'agit d'une philosophie qui s'épanouit grâce à la destruction et à l'assujettissement de peuples non-européens.

Tandis que la foule paysanne française exulte suite à l'instauration des cahiers de doléance, Zéphyr commente cyniquement : " Laissons-les à leur joie ! Ils n'ont pas si souvent l'eau du bonheur dans les yeux ! Chez nous, à la Guadeloupe, ce mois de mai 1789 est pareil a tous les autres. Sans soleil, sans joie ! » (21). Sans transition, une didascalie indique que « le podium de gauche s'éclaire. Un groupe d'esclaves regarde un grand nègre, entouré de deux contremaîtres. » La scène de châtiment corporel qui succède 
rappelle ironiquement que ce qui vaut pour la France (franco-française, blanche et métropolitaine) ne vaut pas pour les peuples noirs des Antilles. Voyons une autre scène :

LA VOIX : Les hommes naissent et demeurent libres et égaux en droits.

Les esclaves se jettent dans les bras les uns des autres, pleurent, s'embrassent.

LES ESCLAVES : Liberté ! Liberté !

MONSIEUR DE JUREMONT : Taisez-vous ! IL ne s'agit pas de vous. On a parlé des hommes. Pas des nègres ! (31)

L'incorporation de passages de la Déclaration des droits de l'homme et du citoyen ainsi que de retranscriptions de débats à l'Assemblée nationale de l'époque produit un pastiche savoureux. Il est évident qu'au cœur d'une société esclavagiste et coloniale, ces beaux discours apparaissent pittoresques et participent de l'atmosphère carnavalesque et absurde d'ATR. Condé invite le spectateur guadeloupéen (et domien plus généralement) à réfléchir au « deux poids, deux mesures » qui a de tout temps prévalu dans les relations entre la France et ses colonies et départements. En effet, même après la départementalisation (pensée initialement pour neutraliser le statut colonial de ces régions), les DOM ont continué à être ces éternelles exceptions au sein du territoire français en ne bénéficiant pas des mêmes droits et avantages que leurs homologues métropolitains. Par conséquent, l'ironie est également largement utilisée par Condé dans un but pédagogique.

Dans son livre Les Misères des Lumières : Sous la raison, l'outrage..., Louis Sala-Molins a largement dénoncé un bicentenaire qui s'accommodait parfaitement du paradoxe des Lumières et qui, en outre, occultait sans vergogne la Révolution haïtienne, jugée par l'historien comme étant la seule vraie réussite de la pensée des Lumières. En effet, la Révolution haïtienne porta à leur radicale interprétation les notions d'universalité, d'humanité et de fraternité que la philosophie des Lumières prétendait défendre. L'historien dénonce l'hypocrisie institutionnalisée d'un État qui préfère maintenir dans la périphérie des évènements en réalité centraux au regard de la période révolutionnaire, en vue d'exercer des rapports de pouvoirs néocoloniaux. Il conclut que si la Révolution et les Lumières sont si bien commémorées par la France socialiste des années 1980-1990, c'est parce que cette dernière se reconnaît dans cette pensée « éclairée » riche en discours grandiloquents mais pauvre en résultats. Toutes deux ont abandonné leurs projets éthiques (émancipation des esclaves pour la première et justice sociale pour la seconde).

\section{Dans Staging Creolization: Women's Theater and Performance from the French Caribbean, Emily} Sahakian suggère que ce trope des espoirs déçus et des promesses non tenues se cristallise avec la métaphore de l'enfant à naître (« unborn child») personnifié à travers le personnage de Solitude. Elle explique :

In conceiving of the fruits of revolution as an unborn child, An tan revolisyon bridges the conventions of postcolonial and intercultural theaters. Condé does not foreclose the possibility of a universalist revolution to come, but she does show how the allegedly universalist ideals of the Revolution were never meant to benefit people in the Caribbean. (63)

Sur ce point précis, Sala-Molins nous gratifie d'une formule édifiante : « De la Révolution, nous en avons carrément gommé la révolution. » (211).

$A T R$ est sans conteste la mise-en-scène des espoirs déçus et de la trahison du langage universaliste. Cependant, la dramaturge prend garde de se prémunir contre « une mise en schéma » et « un pleur nostalgique » (Glissant 226). Autrement dit, il ne s'agit pas de pleurer sur son sort (ou plus exactement le sort des ancêtres) et de tomber ainsi dans une logique de victimisation. Il faut aborder une vision futuriste du passé, non un passéisme immobilisant. C'est ainsi que Glissant conçoit sa vision prophétique du passé 
: «C'est à démêler un sens douloureux du temps et à le projeter à tout coup dans notre futur, sans le recours de ces sortes de plages temporelles dont les peuples occidentaux ont bénéficié, sans le secours de cette densité collective que donne d'abord un arrière-pays culturel ancestral. C'est ce que j'appelle une vision prophétique du passé. » (226-227).

C'est dans cette perspective que Zéphyr assume la responsabilité de son rôle d'éclaireur :

« Je suis fatigué. Je voudrais retourner dans le ventre de ma mère et m'y blottir comme un mâle-crabe au fond du trou dans la terre. Je voudrais fermer les yeux et mes oreilles et ne plus rien entendre des bruits de notre monde. Je voudrais mourir une deuxième fois. Hélas, je dois vous conduire au terme de ce voyage à rebours du temps. Même si cela me met la bouche en sang et les yeux en eau, je suis bien obligé d'aller avec vous jusqu'au bout. » (Condé 68)

Stéphanie Bérard explique que la culture antillaise est fondamentalement théâtrale et parmi les nombreuses traditions populaires caribéennes, le carnaval, le vaudou et le gwoka (tambours) sont les plus à même d'inspirer les dramaturges en tant que pratiques liées à la musique et à la danse (153). Ces trois pratiques se retrouvent dans la pièce de Condé et vont ainsi parvenir à cannibaliser et " caribéaniser » l'Histoire. Dans ses indications scéniques, Condé mentionne que le mode de jeu doit renvoyer au style de la foire populaire et a toutes les formes de théâtres de rue. Le « mode bouffon » est le plus prisé, mais on y ajoute également la farce et le mime par le biais des marionnettes, des clowns, des saltimbanques. La scène se transforme en véritable carnaval.

Le premier personnage qui entre en scène (après le conteur) donne immédiatement le ton non réaliste de la représentation où la performance (qu'elle soit acrobatique, artistique, comique, etc.) détourne le « sérieux » du sujet historique. La didascalie précise : « Un Blanc richement vêtu passe, fait la roue et disparaît. » (Condé 18) Le roi mais également ses conseillers (députés) apparaît comme de parfaits pantins ridicules de comique : « Le podium de droite s'est éclairé. Sous les yeux du roi, toujours caricaturalement assis sur son trône, qui d'abord tente de les arrêter, puis se bouche les oreilles, puis se cache le visage, les députés se lèvent, se rassoient comme des marionnettes, débitant très vite, de plus en plus vite, avalant. » (29).

Monarchie et République sont renvoyées dos à dos; car aucun de ces régimes ne se soucie du sort des esclaves noirs qui demeurent dans leur servitude dans les lointaines colonies. À travers ce spectacle haut en couleur, Condé dénonce l'illusion révolutionnaire et droit-de-l'hommiste de 1789 comme conclut Zéphyr à la fin de la première partie : « [...] Pour nous autres, en Guadeloupe, pas grand-chose de changé. Nos hommes, nos femmes continuent d'avoir à la bouche le goût de la liberté. Hélas, ils sont enchaînés et nos morts continuent de saigner leur sang dans les silos de la terre avant que leurs esprits ne volètent au serein dans le corps des bêtes à feu. » (36).

\section{PLAIDOYER POUR UNE RADICALE COOPÉRATION CARIBÉENE}

Pour la dramaturge, l'histoire ne s'arrête pas aux frontières métropolitaines mais se joue aussi et surtout sur d'autres théâtres géopolitiques. Les trois aires représentent donc les trois zones géographiques dont il est question dans la pièce : la Guadeloupe, Haïti et la France. Par sa mise en scène, Condé met en perspective la relation glissantienne à travers une toile de connexions historico-géographiques. Glissant explique : "Là où se joignent les histoires des peuples, hier réputés sans histoire, finit l'Histoire. (Avec un

Alternative francophone

https://journals.library.ualberta.ca/af/index.php/af 
grand H.) L'histoire est un fantasme fortement opératoire de l'Occident, contemporain précisément du temps où il était le seul à 'faire' l'histoire du monde. » (Le Discours antillais 227). Et un peu plus loin il ajoute : «A ce stade, l'Histoire s'écrit avec un grand H. C'est une totalité qui exclut les histoires non concomitantes de celle de l'Occident. » (Le Discours antillais 243). Ces deux citations ont le mérite d'expliquer autant que d'apporter des solutions à ce manque d'historiographie : c'est aux peuples caribéens d'écrire leurs histoires face à l'Histoire.

La transversalité est le remède préconisé par Glissant pour s'affranchir du joug de la colonialité : les pays caribéens doivent écrire leurs histoires transversales contre la verticalité des rapports dominant/dominé. La transversalité est le pendant historique de la théorie rhizomatique glissantienne. En écrivant leurs histoires enchevêtrées, les peuples de la Caraïbe s'envisagent les uns par rapport aux autres et entrent dans la Relation émancipatrice comment suggéré ici : « se battre contre l'Un de l'Histoire, pour la Relation des histoires, c'est peut-être à la fois retrouver son temps vrai et son identité : poser en termes inédits la question du pouvoir. » (Le Discours antillais 276).

Dans Mémoire des esclavages, Glissant peaufine sa notion de transversalité en proposant que celle-ci a pour but ultime de révéler l' « histoire cachée » :

[...] relevant d'une même indiscernable poussée de ces histoires, désignent à nos yeux des mouvements qui convergent en s'ignorant, alors même que ces figures ne révèlent en aucune manière qu'il y aurait, ou qu'il pourrait y avoir, des conséquences semblables à souligner dans les lieux différents et autonomes, apparemment, de ces histoires, ou des conséquences contraires à révéler dans des circonstances semblables, les continuités dites logiques sont camouflées partout, c'est ce qu'on appellerait une histoire cachée, ou une histoire qui se dit sans se dire tout en disant." (Mémoire des esclavages 56)

ATR se clôt sur la représentation de cette transversalité non sans une certaine ironie tragique (quand on connaît le traitement fait aux immigrés haïtiens en Guadeloupe) :

DESSALINES : Ne pleure pas! Est-ce que tu n'es pas ici chez toi ? Est-ce que la Guadeloupe et SaintDomingue, ce n'est pas pareil?

LA FOULE : C'est pareil! C'est pareil!

DESSALINES : Est-ce que ce n'est pas d'Afrique que nous sortons tous?

LA FOULE : Oui! Oui!

DESSALINES : Est-ce que ce n'est pas le même créole que nous parlons?

LA FOULE : Oui! Oui!

DESSALINES : Est-ce que ce n'est pas le même tambour que nous dansons?

Battements de tambour.

LA FOULE : Oui! Oui!

DESSALINES : Guadeloupéens, Guadeloupéennes, ne pleurez pas! Vous êtes ici chez vous! Cette terre, c'est la vôtre! Nous la partageons avec vous et si vous étiez des milliers à nous demander refuge, nous vous accueillerions de la même façon!

Ovation. Tambour. Applaudissements. (79-80)

Notre recul contemporain ne peut que constater l'inexistence d'une telle fraternité pancaribéenne, comme en témoignent les échecs successifs d'une confédération antillaise ou autre projet d'avenir commun. Bien que la pièce se dérobe constamment à l'univocité de son message, elle entrouvre quelques pistes à explorer...

Les trois espaces et les trois périodes qui structurent la pièce nous font nous interroger sur la symbolique de ce nombre. À ce propos, dans l'article intitulé « Protean Truths: History as Performance in Maryse Condé's An Tan Revolisyon », Deborah Gaensbauer cite Jane Plastow : "Caribbean history is by 
definition spatial, haunted by echoes of the Atlantic Triangle, the poignant metaphorical voyage "home" in search of the self, for which Césaire's Cahier d'un retour au pays natal is the ultimate paradigm." (1145). Toutefois, on note la disparition de l'Afrique dans le triangle tel que le redéfinit Condé. En effet, la dramaturge recentre son histoire/Histoire de l'autre côté de l'Atlantique et s'intéresse à valoriser les liens historiques et géographiques qui unissent la Guadeloupe et Haïti (et par extension toutes les îles de la Caraïbe).

Il serait simplificateur d'interpréter cette absence par le rejet pur et simple de l'Afrique (car l'Afrique plane en toile de fond culturelle et spirituelle tout au long de la pièce); il s'agit plutôt de la part de l'auteure de refuser un mythe des origines. De la même manière que la pièce s'éloigne - en le parodiant du mythe de la Révolution française, l'Afrique ne peut fonctionner comme le catalyseur de toutes les frustrations des peuples antillais. L'idée d'une identité-racine d'un peuple unique souscrivant aux valeurs d'un récit mythique des origines est résolument abandonnée dans cette vision des choses. En cela, Condé rejoint pleinement Glissant qui souhaitait lui aussi revenir au « pays réel » comme le souligne le critique Michael Dash dans son ouvrage Edouard Glissant: "With the 'pays légal' official in the hands of the French, and the 'pays rêvé' of Africa lost forever, Glissant looked to the 'pays réel' as the space that needed to be investigated and from which solutions had to be derived. » (92).

Comme l'a souligné Mackward, Condé a décrété la guerre aux idées convenues et s'en en souvent prise au colorisme, au négritudisme et nombre d'autres -ismes. (40).

Dans la pièce, Zéphyr dénonce ces dangereux essentialismes tels que le noirisme ou le duvaliérisme en tant que dérives fascisantes : «Qu'est-ce qu'il penserait, Toussaint, le vieux Toussaint, s'il revenait à la vie et voyait le pays à l'encan, livré à la rage des Tontons Macoutes, son peuple saignant des éternelles blessures de l'injustice et de la faim ? Qu'est-ce qu'il ferait en s'apercevant que les ennemis des Noirs ne sont pas seulement les Blancs? »(38-39).

En tant que victoire sur l'esclavage et le colonialisme, Sahakian rappelle la façon dont Haïti (et sa révolution) ont toujours fasciné les auteurs antillais. Les auteurs des années 1960 et 1970 sont enclins à la description de ces grandes figures historiques telles que Toussaint Louverture et Henri Christophe. Par contraste, les écrivaines antillaises montreront dans les années suivantes un vif intérêt pour décrire Haïti aussi bien dans sa dimension passée que présente afin de souligner « the triumph of the Haitian Revolution while also valorizing the daily lives of ordinary people and recognizing Haiti's ongoing struggle. » (7).

\section{UNE CEUVRE POUR LA MÉMOIRE, CONTRE LA COMMÉMORATION}

Dans un entretien, Condé confia à Nick Nesbitt que certains historiens guadeloupéens avaient rudement critiqué sa pièce pour avoir « dépeint Toussaint Louverture sous les traits d'un dictateur » (62). Il est vrai que l'atmosphère bouffonne définit l'intégralité de l'univers d'ATR et les héros noirs n'échappent pas à cette irrévérence systématique. Les héros antillais subissent un traitement qui fut très critiqué par les fétichistes de ces figures historiques (notamment dans les milieux nationalistes). La pièce malmène particulièrement Toussaint Louverture qui, entre autres, est présenté comme une pop-star mégalomane

Alternative francophone

https://journals.library.ualberta.ca/af/index.php/af 
entourée de ses groupies. Le culte de la personnalité ajouté au caractère tyrannique excessif de Toussaint Louverture crée un décalage qui provoque une scène extrêmement comique. Les didascalies indiquent :

«Le podium 1 s'est allumé. Toussaint Louverture ovationné par les siens et applaudi par les généraux blancs, va s'asseoir sur une sorte d'estrade et tient audience.»

UN HOMME (se jetant à ses pieds) : Général, Sauveur, Osagyefo, Premier des Noirs, Mwalimwana, je viens du Mirabalais. L'Habitation que tu as bien voulu me louer de donne plus rien, ni sucre, ni café. Depuis qu'ils sont libres, les nègres ne veulent plus rien faire.

TOUSSAINT LOUVERTURE : Comment cela? Le travail est une vertu. Tout homme errant et oisif sera arrêté et puni par la loi. Fais-toi aider par le commandement militaire de ta région.

UN SOLDAT (poussant devant lui un groupe d'hommes.) : General, Sauveur, Osagyefo, Mwalimwana... TOUSSAINT LOUVERTURE : Abrège!

UN SOLDAT : Ces paysans viennent de Dondon. Ils se sont soulevés.

DES VOIX DANS L'ASSISTANCE : Ceux de Marmelade, de Plaisance et de Limbé aussi.

TOUSSAINT LOUVERTURE : Qu'est-ce qu'ils veulent encore? Est-ce qu'ils ne sont pas libres?

UN SOLDAT : Abrège, je t'ai dit! Tu ne m'as pas entendu?

UN SOLDAT : Oui, Sauveur, j'abrège! Ils veulent le partage des terres!

TOUSSAINT LOUVERTURE : (furieux.) Quoi?

LE SOLDAT : Le partage des terres, Petit Père des Peuples! La terre à ceux qui la cultivent. Ils disent que tes généraux ont fait main basse sur les propriétés des Blancs et qu'ils ne sont pas mieux lotis que du temps de l'esclavage. Ah non, pas mieux du tout!

TOUSSAINT LOUVERTURE : Quelle insolence! Qu'on leur donne le fouet!

Il se lève.

TOUSSAINT LOUVERTURE : (vociférant.) Tous les gérants, conducteurs et cultivateurs qui ne rempliront pas avec assiduité les devoirs que leur impose la culture, seront arrêtés et punis avec la même sévérité que les Militaires qui s'écartent des leurs... J'ai dit, moi, Toussaint Louverture, général en chef des armées.

UN AUTRE SOLDAT : (s'avançant avec un groupe de femmes.) Père. Bienfaiteur, ces femmes disent que...

TOUSSAINT LOUVERTURE : Tais-toi. Je ne veux plus rien entendre. L'audience est terminée.

Il se lève et se retire. L'assistance le suit. La lumière s'éteint. (49-50)

Ce que n'ont pas su lire entre les lignes les détracteurs de Condé est le fonctionnement métonymique du père de la Révolution haïtienne. En nous présentant Toussaint comme un personnage qui s'est coupé de la révolution populaire, c'est en réalité le concept de révolution lui-même qui est remis en cause. Toussaint symbolise la révolution (et ses lendemains désenchantés) et pour cela il est passablement malmené. Zéphyr résume sa pensée en ces mots: «Et voilà la révolution, c'est comme une femme, on en fait ce qu'on veut. Les soudards la sodomisent. Les poètes lui récitent des vers. Les bourgeois lui font cracher des sous. »(35).

On remarquera en outre que les doléances des femmes sont encore moins dignes d'importance puisque la séance s'interrompt brusquement sans qu'on ne juge bon de les recevoir. En outre, Zéphyr renchérit en interpellant le public : «Eh là, eh là ! Il m'a fait un peu peur, à moi Toussaint Louverture, malgré tout le respect qu'on lui doit. Pas à vous ? » (50). En effet, la démythification systématique des héros antillais a également pour but de repenser le rôle des anonymes dans cette histoire oubliée, et en particulier celui des femmes.

Ainsi, le second héros antillais démystifié par la dramaturge n'est autre que Louis Delgrès, qui brille par son absence. Il est connu pour être le meneur des Noirs insurgés qui s'opposèrent aux troupes 
napoléoniennes venues rétablir l'esclavage en Guadeloupe en 1802. Nous interprétons ce «manque » délibéré comme la volonté de l'auteure de refuser la mythification phallocentrique de l'histoire. La seule occurrence où est mentionné le nom de Delgrès tourne très rapidement à la scène comique :

IGNACE : Nous allons rejoindre les forces du commandant de la Basse-Terre, Delgrès et ensemble, nous déferons les ennemis de notre liberté.

Hurlement de la foule.

UN AUTRE OFFICIER : (prenant place sur l'estrade.) Citoyens, je vais vous donner lecture de la proclamation du commandant de la Basse-Terre Delgrès.

(il lit très mal.)

" À l'univers entier. Le dernier cri de l'innocence et du désespoir. C'est dans les plus beaux jours d'un siècle à jamais célèbre par le triomphe des lumières et de la philosophie ».

La foule commence visiblement à s'agiter.

L'OFFICIER : ... qu'une classe infortunée qu'on veut anéantir se voit obligée d'élever la voix vers la postérité...

LA FEMME : Qu'est-ce que cela veut dire?

UN HOMME : Tais-toi! C'est du français-français.

UN AUTRE : Vos gueules! Tant pis pour vous, si vous ne comprenez pas le français! (72)

Cette parodie de discours nous entraîne bien loin de la commémoration solennelle; car pour Condé la récupération de l'histoire antillaise ne doit pas être synonyme de glorification de héros tutélaires. Dans cette scène, à l'instar de celle reproduite précédemment, on constate que la parole des femmes est systématiquement reléguée au second plan. Ces grandes figures héroïques masculines empêchent de reconnaître à sa juste valeur la participation du petit peuple d'une part, mais aussi et surtout celui des femmes, dont le rôle ne fut que très rarement retenu par l'historiographie officielle.

L'année 1952 voit la consécration de Louis Delgrès comme héros national guadeloupéen. Le journal communiste $L$ 'Etincelle entreprend une systématique revalorisation du personnage qui devient un « martyr » représentant les masses exploitées. Nesbitt évoque une idéologie totalisante de Delgrès très en accord avec l'époque. Delgrès est depuis lors un symbole manipulé, muséifié, commémoré religieusement par les élites locales qui le récupèrent habilement. L'histoire - sa commémoration - devient alors un spectacle, un produit comme un autre dans cette (pour reprendre les mots de Nesbitt) : « radically static vision of a Guadeloupe frozen into a world of commodification. » (74).

Dans La Mémoire, l'histoire, l'oubli, Paul Ricœur développe le concept de « mémoire manipulée » (partie de la triade «mémoire empêchée », « mémoire manipulée » et «mémoire obligée »). Selon lui, la mémoire manipulée se situe à l'intersection entre le problème de l'identité et celui de la mémoire. C'est à ce moment précis que « la ressource du récit devient ainsi le piège, lorsque des puissances supérieures prennent la direction de cette mise en intrigue et imposent un récit canonique par voie d'intimidation ou de séduction, de peur ou de flatterie. » (580).

Dans la réplique qui ouvre la pièce, Zéphyr annonce : « Il faut donc que je vous rafraîchisse la mémoire et que je vous rappelle cette histoire dont vous n'avez plus le souvenir. » (17). Tout en contribuant à la restauration d'un passé négligé, Condé parvient habilement à ne pas tomber dans le piège de la mémoire manipulée, autrement dit, de la commémoration (qu'elle soit nationaliste/guadeloupéenne ou républicaine/métropolitaine). De fait, Nesbitt rappelle que Delgrès fut récupéré dès le XIXe siècle par le

Alternative francophone

https://journals.library.ualberta.ca/af/index.php/af 
discours républicain. Le roman historique, Le chasseur de rats : le colonel Delgrès écrit en 1876 par Gustave Aimard est une parfaite illustration du projet colonialiste amorcé par la République en cette fin de siècle. Malgré ses préjugés racistes, Aimard dépeint Delgrès comme le valeureux républicain qui se rebella contre la tyrannie napoléonienne.

L'historien américain Laurent Dubois dans son article « Haunting Delgrès » souligne « the ambiguities of celebrating Delgrès, a male hero who chose to commit suicide in a great gesture, while generations of forgotten women struggled in other and perhaps more powerful ways against the degradation of slavery. » (176). Une interprétation similaire se retrouve chez de nombreux critiques et notamment chez Dash qui explique - concernant le choix de Glissant d'écrire sur Louverture plutôt que sur Delgrès—:

It is perhaps significant that Glissant chose to write a play about Toussaint and not someone like General Louis Delgrès who, with his soldiers, committed mass suicide in Fort Matouba rather than surrender to General Richepanse, who had been sent to restore slavery in Guadeloupe. This romantic act of selfimmolation is less interesting to Glissant than Toussaint's attempt to live through the tragic consequences of his ideological choices. (106)

Le choix de ne pas sacraliser Delgrès suggérerait-il un postulat idéologique quant à la solution du suicide collectif qui apparaît comme une fausse alternative? L'interprétation plurivoque de l'œuvre ne permet pas d'avancer de réponse... Si l'on transpose cela dans le contexte de forte agitation indépendantiste des années 1980, en faisant le choix de ne pas représenter Delgrès, Condé refuserait-elle cette attraction qui pousse les jusqu'au-boutistes nationalistes à préférer l'autodestruction à la colonisation? Rosello questionne également cette posture élitiste : « cette supposition ne revient-elle pas à condamner tout un peuple à mort au nom de sa libération éventuelle? N'y a-t-il pas simplification grandiloquente et outrancière à supposer que le peuple colonisé en sera réduit au suicide s'il veut sortir de l'asservissement où le confine le colonisateur? L'anéantissement de tout un peuple n'est-il pas au contraire la conséquence la plus à craindre de la colonisation? » (43-44).

En réponse à ses actions viriles performées pour la postérité, la dramaturge choisit de valoriser la femme dont le témoignage a disparu. Ainsi, Solitude est l'unique personnage qui échappe à l'ironie et au mode bouffon. On lit les indications scéniques : «Évidemment, cette époque est moins bouffonne que les deux premières. Néanmoins, elle ne doit jamais être jouée tragique. Au contraire, très sec. Les moments parodiques doivent être appuyés. À l'exception de la courte scène entre Solitude, Joséphin et Sergelius, il ne doit y avoir aucune émotion » (59).

Zéphyr symbolise peut-être une alternative viable à la sur-masculinisation du politico-historique aux Antilles. Conçu par l'auteure comme une figure ambiguë (y compris en ce qui concerne son genre), son jeu d'acteur devait mettre l'accent sur son côté féminin. Condé, qui ne s'est jamais considérée comme féministe, - ici entendu dans le sens discriminatoire et non-inclusif d'une définition étroite du terme-a toujours souhaité la collaboration entre hommes et femmes. En 1982, elle expliquait que « la femme antillaise ne considère pas l'homme comme l'adversaire, encore moins comme l'ennemi, mais comme une victime, au même titre qu'elle, de la société esclavagiste et du pouvoir colonialiste. L'homme et la femme ne sont pas en guerre entre eux. » (Kamal 150).

De cette manière, Zéphyr incarne le devenir collectif du peuple guadeloupéen (et caribéen par extension) chez qui il tente d'éveiller la conscience historique. À la fin, le choix appartient au spectateur/lecteur de 
tirer sa conclusion, sa propre interprétation de l'histoire et de faire le rapprochement avec la situation actuelle et celle qu'il projette pour le futur :

C'était en 1802! Puisque nous connaissons tous la suite de cette histoire, je vous laisse juger si les promesses faites cette année-là ont été tenues, si les rêves ont été réalisés ou si demain reste encore à naitre, tapi dans le ventre de votre mère. Mes yeux a moi ne voient rien; que des morts, beaucoup, beaucoup; des prisonniers, beaucoup, beaucoup, des exilés, beaucoup, beaucoup... Il se fait tard. Il est temps que je retourne à ma nuit éternelle, à ma couche sous les filaos, à deux pas de la mer! La mer, c'est ma compagne. [...] Je vous rends à la vie! Au soleil! Aux fleurs de balisiers, rouges au flanc du volcan. (81)

\section{CONCLUSION}

An tan revolisyon est une œuvre vitaliste qui transmet un message très fort aux peuples antillais en ce qui concerne leur aliénation vis-à-vis de la métropole et un immobilisme politique. Le théâtre intellectuellement et philosophiquement polyvalent de Condé propose une voie/voix alternative à l'historiographie traditionnelle, en particulier sur la période révolutionnaire. Si la Révolution de 1789 est immédiatement évacuée comme mythe fondateur de la nation française (puisque le discours officiel n'a pas pris en compte la réalité des peuples antillais et préféra taire leurs histoires transversales), la dramaturge ne le substitue aucunement par un mythe nationaliste guadeloupéen (ou caribéen) ainsi qu'en atteste le traitement réservé aux héros de la Caraibe. La perspective féminine, l'esthétique carnavalesque et la voix du conteur créole contribuent à réévaluer la valeur du mythe en tant qu'histoire sacrée.

Enfin, cette pièce invite le spectateur antillais à se projeter dans le futur par le biais de l'imagination historique en jouant un rôle actif dans l'élaboration de nouvelles potentialités. Cette pièce n'est pas programmatique; en cela aucune solution explicite n'est à attendre du théâtre de Condé. En bref, elle propose et le public dispose... Trente ans après sa première représentation, cette « tragédie optimiste » (pour reprendre le terme de Makward) est encore et toujours d'actualité comme les évènements de mars 2017 en Guyane nous l'ont démontré.

Alternative francophone

https://journals.library.ualberta.ca/af/index.php/af 


\section{BIBLIOGRAPHIE}

Bérard, Stéphanie. Théâtres des Antilles : Traditions et scènes contemporaines. L'Harmattan, 2009.

Bonilla, Yarimar. Non-sovereign Futures: French Caribbean Politics in the Wake of Disenchantment. University of Chicago Press, 2015.

Carruggi, Noëlle. Maryse Condé: Rebellion et transgressions. Paris, Khartala, 2010.

Certeau (de), Michel. L'Ecriture de l'histoire. Gallimard, 2011.

Condé, Maryse. An Tan Révolisyon: Elle Court, Elle Court La Liberté. Editions de l'Amandier, 2015.

Dubois, Laurent. "Haunting Delgrès." Radical History Review, vol. 78, 2000, pp. 166-177.

Fischer-Lichte, Erika. The Routledge Introduction to Theatre and Performance Studies. Routledge, 2014.

Gaensbauer, Deborah B. "Protean Truths: History As Performance in Maryse Conde's An tan revolisyon". The French Review, vol. 76 no. 6, 2003, pp. 1139-1150.

Glissant, Édouard. Le Discours Antillais. Gallimard, 2012.

—. Mémoire des esclavages. Gallimard, 2007.

Makward, Christiane. «L'ironie comme stratégie dramatique dans "la tragédie du Roi Christophe" d'Aimé Césaire et "An Tan Revolisyon" de Maryse Condé. » Francofonia, no 61, 2011, pp 109-123.

Nesbitt, Nick. Voicing memory: History and Subjectivity in French Caribbean Literature. University of Virginia Press, 2003.

Ricœur, Paul. La mémoire, l'histoire, l'oubli. Seuil, 2002.

Rosello, Mireille. Littérature et identité créole aux Antilles. Karthala, 1992.

Sahakian, Emily. Staging creolization: Women's theater and performance from the French Caribbean. University of Virginia Press, 2017.

Sala-Molins, Louis. Les misères des Lumières : sous la raison, l'outrage. Homnisphères, 2008.

Salhi, Kamal. Francophone Voices. Elm Bank Publications, 1999. 